\title{
Simulazione della propagazione di difetti a fatica mediante il modello di zona coesiva
}

\author{
A.Pirondi, F.Moroni \\ Università degli Studi di Parma, Dipartimento di Ingegneria Industriale, v.le G.P. Usberti 181/ A, 43100 Parma; \\ alessandro.pirondi@unipr.it
}

RiASSUNTO. Le giunzioni incollate guadagnano sempre più mercato, nel campo delle costruzioni in genere, dove è necessario un alleggerimento delle strutture. Nel caso di geometrie di giunto semplici il dimensionamento avviene attraverso relazioni analitiche che restituiscono il valore massimo delle tensioni, il quale deve essere inferiore al limite di utilizzo dell'adesivo stesso. Quando le geometrie sono complesse l'approccio analitico diventa impraticabile, di conseguenza si provvede a verificare la correttezza della soluzione mediante analisi agli elementi finiti (EF). L'introduzione del modello di zona coesiva nell'analisi EF permette di simulare il danneggiamento ed il cedimento del giunto in condizioni quasi-statiche e impulsive. In questo articolo si vuole implementare il modello per la simulazione della propagazione a fatica di difetti, utilizzando il software agli elementi finiti ABAQUS assieme a subroutine esterne interagenti con il modello EF stesso. Un punto focale dell'implementazione sarà il calcolo automatico del tasso di rilascio di energia $G$ in modo indipendente dalla geometria del difetto stesso. I parametri del modello ricavati da prove di tenacità a frattura e propagazione di difetti a fatica in modo I, saranno utilizzati come riferimento per la convalida dell'implementazione.

ABSTRACT. This work deals with the prediction of the fatigue crack growth of bonded joints. Traditionally cohesive zone model is used for the simulation of failure of bonded joint under quasi-static and impact conditions. On the other hand fracture mechanics concepts are used for the prediction of fatigue crack growth in bonded joint. In this work a modified cohesive damage model, accounting for the fatigue crack growth, was implemented in the commercial software ABAQUS. The fatigue damage evolution in the cohesive element is related to the number of cycles through the experimental fatigue crack growth behavior. An automated procedure, valid for any from the geometry and load distribution, was also defined to compute the Strain Energy Release Rate, the necessary input to define the instantaneous fatigue crack growth rate. The model is validated by comparing the simulation to fatigue crack growth tests.

Parole Chiave. FEM; Modello di zona coesiva; Giunti incollati; Fatica; Propagazione difetti.

\section{INTRODUZIONE}

I fenomeni che portano un giunto incollato al collasso possono sia essere dovuti a carichi quasi statici che a carichi affaticanti. A seconda dei casi, le metodologie utilizzate per la previsione della resistenza del giunto possono consistere in: i) previsioni basate su modelli analitici; ii)simulazioni agli elementi finiti (EF). In entrambi i casi si può valutare quindi se riferirsi semplicemente alle tensioni nell'adesivo o se adottare un approccio più generale di meccanica della frattura. In quest'ultimo caso, la simulazione EF con il modello di zona coesiva $[1,2]$ è un metodo efficace per la previsione di cedimento in condizioni quasi-statiche e impulsive [3 - 11].

In particolare il modello di zona coesiva prevede un legame tra le tensioni che si possono avere in prossimità dell'apice del difetto e l'apertura e/o scorrimento dello stesso. Questo modello di comportamento può essere mutuato per simulare il danneggiamento legato a fenomeni di fatica [12-17]. Nella maggior parte di questi lavori i parametri del modello vengono 
opportunamente tarati in modo da riprodurre prove di propagazione a fatica di difetti, mentre in [12] la degradazione a fatica della zona coesiva è legata direttamente al rateo di avanzamento ciclico del difetto attraverso un modello di omogeneizzazione del danno (transizione micro-macroscala). Il limite del lavoro effettuato in [12] è rappresentato dalla mancanza di generalità nel calcolo del valore del tasso di rilascio di energia, $G$, da cui dipende la velocità di avanzamento del difetto. In questo articolo si vuole implementare un modello per la simulazione della propagazione di difetti a fatica basato sul lavoro presentato in [12]. L'implementazione sfrutterà il software agli elementi finiti ABAQUS attraverso l'utilizzo di subroutine esterne interagenti con il modello EF stesso. Un punto focale dell'implementazione sarà il calcolo automatico di $\mathrm{G}$ in modo indipendente dalla geometria del difetto stesso. I parametri del modello ricavati da prove di tenacità a frattura e propagazione di difetti a fatica in modo I, saranno utilizzati per come riferimento per la convalida dell'implementazione.

\section{ASPETTI TEORICI}

$\mathrm{I}$

1 modello di zona coesiva è un modello ampiamente studiato e utilizzato, in cui la frattura viene descritta come un fenomeno di progressiva separazione lungo una porzione di materiale, detta appunto zona coesiva, nella quale agiscono trazioni all'interfaccia delle superfici di frattura, come schematicamente mostrato in Fig.1.

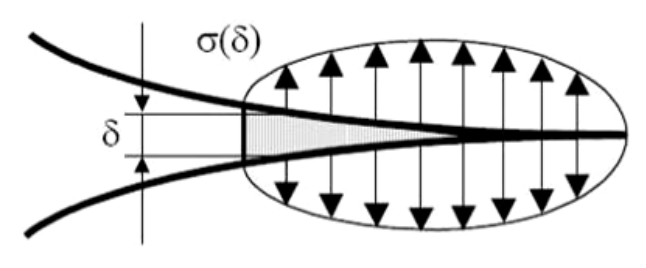

Figura 1: Modello di zona coesiva.

Si può quindi definire un legame tra l'apertura del difetto e la tensione esercitata, come ad esempio riportato in Fig. 2.

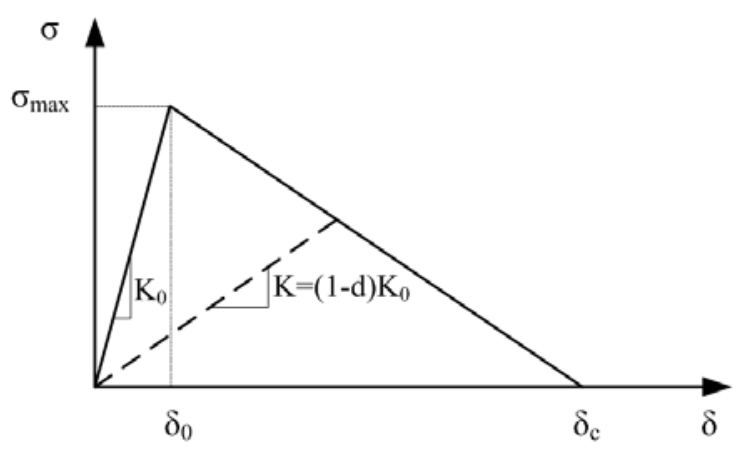

Figura 2: Legame tensioni-apertura per il modello di zona coesiva.

La prima parte è puramente elastica, mentre superato $\delta_{0}$, il comportamento è caratterizzato da un danno $d$ che agisce degradando la rigidezza rispetto a quella iniziale, fino a $\delta_{C}$ in cui non viene esercitata più alcuna tensione all'interfaccia. Si può quindi pensare l'interfaccia come una zona in grado di assorbire un energia $\Gamma$ pari all'area sottesa dal triangolo, pari a

$$
\Gamma=1 / 2 \sigma_{\max } \delta_{c}
$$

Questo valore $\Gamma$ rappresenta un energia per unità di superficie ed è sostanzialmente rappresentato dalla tenacità a frattura Gc del materiale. Il modello di zona coesiva è un modello implementato nella maggior parte dei solutori FEM, e risulta essere molto flessibile. Infatti, una volta tarati i parametri per un determinato materiale, questi possono essere utilizzati per qualsivoglia geometria di giunto. 
D'altro canto la vita a fatica può essere analizzata secondo metodologie basate sulla meccanica della frattura e la legge che lega la velocità di avanzamento a fatica del difetto in regime stazionario al carico applicato (legge di Paris [18]) è:

$$
d A / d N=C \Delta G^{m}
$$

in cui $d A$ è l'avanzamento areale del difetto, $\Delta G$ la variazione del tasso di rilascio di energia unitario e $C$ ed $m$ caratteristiche dell'adesivo.

Per utilizzare il modello di zona coesiva nel caso di carichi ciclici, il danneggiamento non deve essere solo legato all'apertura, ma anche alla velocità di propagazione del difetto, funzione della sollecitazione affaticante.

Basandosi su precedenti esperienze degli autori si è scelto di riferirsi ad una semplice legge di tipo triangolare, considerando per il momento il solo modo I. Aumentando l'apertura da una valore nullo, dopo un iniziale comportamento lineare, superato $\delta_{0}$, il modello cerca di rappresentare la formazione di vuoti o microdifetti, fino ad arrivare in corrispondenza di $\delta_{C}$ in cui le superfici di frattura sono completamente formate. Si può quindi definire la perdita di rigidezza in funzione dell'apertura secondo la relazione

$$
d=\frac{\delta_{f}\left(\delta-\delta_{0}\right)}{\delta\left(\delta_{f}-\delta_{0}\right)}
$$

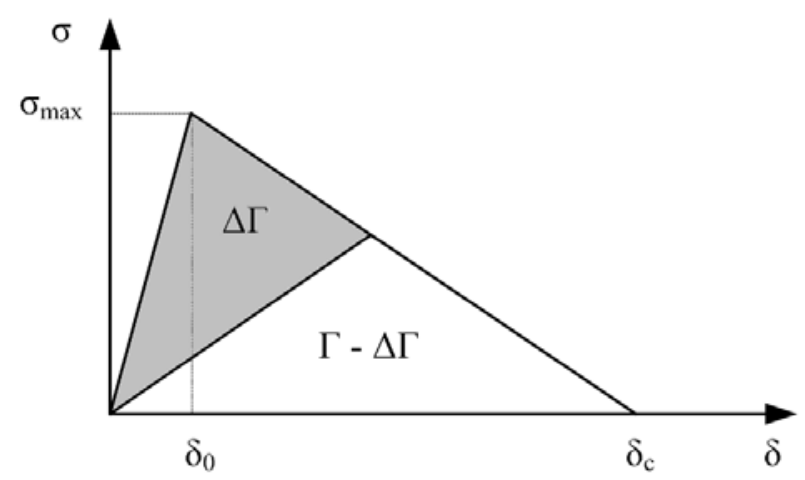

Figura 3: Definizione del danneggiamento in termini energetici.

Secondo quanto postulato in [12], si può quindi definire una seconda variabile di danno $\bar{d}$ che rappresenta il rapporto tra la sezione danneggiata $A d$ e la sezione resistente iniziale $A e$ di un elemento di volume rappresentativo dei processi di danneggiamento (EVR).

Nell'analisi EF Ae coincide con la sezione associata al punto d'integrazione di un elemento coesivo. Questo rapporto può essere legato al rapporto tra l'energia già consumata durante il danneggiamento $(\Delta I)$ e l'energia inizialmente disponibile $(I)$. Ne risulta quindi

$$
\bar{d}=A d / A e^{=\Delta \Gamma / \Gamma}
$$

Attraverso semplici relazioni si può legare il danneggiamento in termini di perdita di rigidezza alla variabile di danno in termini energetici

$$
\bar{d}=\frac{K \delta_{0} \delta_{f}-K \delta_{f} \delta(1-d)}{K \delta_{f} \delta_{0}}=1-\delta / \delta_{0}(1-d)
$$

E sostituendo l'apertura $\delta$ con la sua relazione in funzione del danno $d$ risulta

$$
A d / A e=\frac{d \delta_{0}}{\delta_{f}(1-d)+d \delta_{0}}
$$

Per quel che riguarda la modellazione del danneggiamento a fatica si mettono in relazione grandezze tipiche della meccanica della frattura con il danneggiamento stesso; in particolare si lega la variabile di danno $d$, con l'avanzamento del difetto $d A / d N$. Entrando nel dettaglio la variazione del danno con il numero di cicli può essere espressa attraverso 


$$
d d / d N=d d / d A d d A / d N
$$

dove a sua volta il termine $d d / d A d$ può essere ricavato derivando l'Eq. (6).

$$
d d / d A d=\frac{\left[\delta_{f}(1-d)+d \delta_{0}\right]^{2}}{\delta_{f} \delta_{0} A e}
$$

Il termine $d A d / d N$ invece può essere ricavato formulando un'ipotesi di distribuzione del danno negli EVR dove si sta sviluppando e conoscendo $\Delta G, C$, ed $m$. In particolare, se il giunto viene sollecitato per un numero di cicli $\Delta N$, l'ampiezza della zona danneggiata all'apice del difetto aumenterà di $\Delta A d$, che può essere ritenuto rappresentate ad un avanzamento del difetto equivalente pari a $\Delta A$. L'avanzamento del difetto può quindi essere ritenuto pari alla somma della variazione di area danneggiata di tutti gli elementi che appartengono alla zona di processo $A_{C Z \text {. }}$

$$
d A / d N=\sum_{i \in A c i} d A d^{i} / d N
$$

La zona di processo viene considerata come quella zona in cui avviene il danneggiamento per fatica, ed in particolare è l'area corrispondente a punti di integrazione che hanno un livello di apertura maggiore di $\delta_{t h}$, il quale viene a sua volta determinato conoscendo $\Delta G_{t b}$. In letteratura [12-14], $A_{C Z}$ è stimata attraverso relazioni analitiche, mentre in questo caso sarà automaticamente calcolata come uno dei risultati dell'analisi FEM.

Supponendo che il valore medio della variazione dell'area danneggiata, per gli elementi facenti parte la zona di processo, sia pari a $d A d / d N$, si può riscrivere la relazione precedente come

$$
d A / d N=\sum_{i \in A c Z} d A d^{i} / d N=n_{C Z} d A d / d N
$$

dove $n_{C Z}$ è il numero di elementi che appartengono alla zona di processo. Esplicitando la variazione di area danneggiata con il numero di cicli si ottiene

$$
d A d / d N=\frac{1}{n_{C Z}} d A / d N=\frac{A e}{A c z} d A / d N
$$

dove a sua volta il numero di elementi appartenenti alla zona di processo può essere scritto come il rapporto tra l'area della stessa, Acz, e la dimensione media degli elementi in tale zona $A$ e.

Sostituendo le relazioni trovate nell'Eq. (7) si trova la relazione

$$
d d / d N=\frac{\left[\delta_{f}(1-d)+d \delta_{0}\right]^{2}}{A c z \delta_{f} \delta_{0}} d A / d N
$$

in cui $d A / d N$ è rappresentato dall'Eq. (2).

\section{ASPETTI PRATICI - IMPLEMENTAZIONE NEL SOFWARE EF}

$\mathrm{S}$ i è scelto di implementare la procedura sopra illustrata sfruttando le potenzialità degli elementi coesivi, limitandosi per ora al caso bidimensionale. Il danneggiamento è stato assegnato come una variabile di stato, la quale va ad agire sulla rigidezza del materiale costituente lo strato di elementi coesivi. Inoltre per quel che riguarda tensioni, spostamenti e danno si è sempre fatto riferimento ai punti di integrazione, che nel caso di elementi coesivi bidimensionali sono due. Per valutare l'avanzamento a fatica è necessario, come indicato in precedenza, conoscere il valore di $\Delta G$ durante la propagazione. Questo viene valutato a partire dal calcolo di $G$, a sua volta effettuato secondo la definizione :

$$
G=-\frac{d(W-U)}{d A}
$$

Quindi per pervenire a questo valore è necessario il calcolo dell'energia interna $U$ e del lavoro delle forze esterne $W$, oltre che alla variazione dell'area del difetto $A$. Sebbene questa teoria sia di semplice applicazione il suo utilizzo è reso critico 
dalla valutazione dell'area del difetto. Infatti nei casi in cui la variazione dell'area del difetto, incremento per incremento è ridotta, gli errori numerici vedono i loro effetti amplificati e si possono avere errori sulla valutazione di $G$. D'altra parte variazioni eccessive del danneggiamento incremento per incremento possono condurre ed errori legati alla linearizzazione di relazioni più complesse e anche a problemi di convergenza. A tal proposito si definisce una valore $\Delta \bar{d}$ massimo di incremento del danno $\bar{d}$ che può essere consumato all'interno di un incremento.

Al fine di validare la metodologia che conduce al calcolo di $G$ e conseguentemente di $\Delta G$, si sono eseguiti confronti dell'andamento del tasso di rilascio unitario di energia $G$ con la lunghezza del difetto $a$, per giunti Double Cantilever Beam (DCB), in cui come termine di confronto si sono assunti:

i) la soluzione analitica [19]

$$
G=\frac{(P a)^{2}}{b E I}\left(1+\frac{1}{a \lambda_{\sigma}}\right)^{2}
$$

ii) l'integrale di contorno $J$ valutato medianti analisi FEM lineari elastiche (in (14) $P$ rappresenta il carico applicato, $a$ la lunghezza del difetto, $b$ lo spessore del giunto, $E$ il modulo elastico degli aderendi, $I$ il modulo di inerzia della sezione degli aderendi, $\lambda_{\sigma}$ un fattore correttivo dipendente dalla geometria del giunto e dai materiali in gioco). Il confronto è mostrato in Fig. 4 per un giunto DCB in acciaio con aderendi di lunghezza $120 \mathrm{~mm}$, larghezza $30 \mathrm{~mm}$ e spessore $25 \mathrm{~mm}$ a cui è applicata una forza di $1625 \mathrm{~N}$ avente un difetto iniziale di $45 \mathrm{~mm}$. Si nota come i tre metodi siano praticamente equivalenti per il primo tratto di propagazione.

Oltre ai $15 \mathrm{~mm}$ l'integrale di contorno e il tasso di rilascio di energia unitario si differenziano dall'andamento analitico: questo è prevedibile in quanto l'Eq.14 ha validità solo per condizioni in cui si può considerare l'incollaggio infinitamente lungo (per la geometria a ci si riferisce in Fig. 4 significa $a<60 \mathrm{~mm}$, cioè un avanzamento del difetto $<15 \mathrm{~mm}$ ).

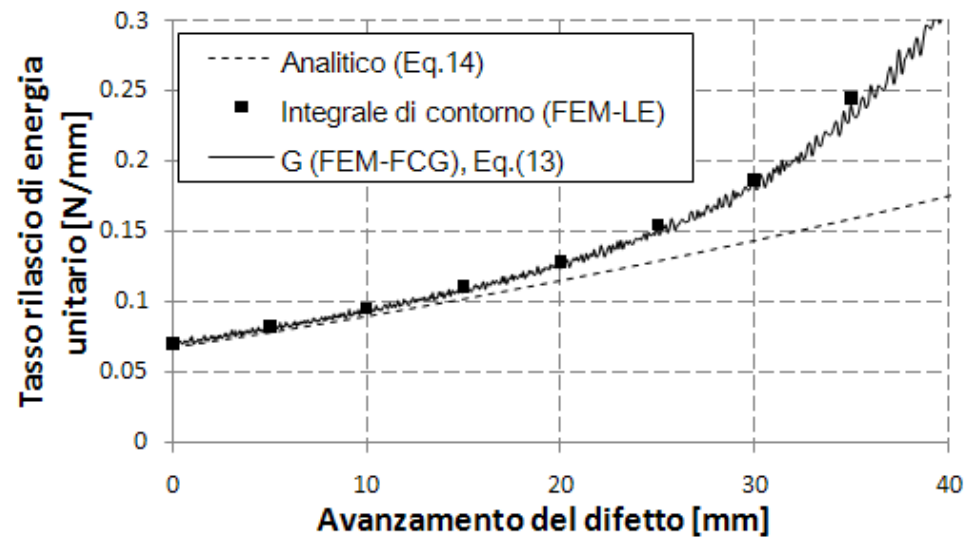

Figura 4: Valori di G calcolati mediate la subroutine e l'Eq. (13) in funzione all'avanzamento del difetto, confrontati con l'andamento analitico (Eq. 14) l'integrale di contorno ottenuto mediante una simulazione FEM lineare elastica.

In ogni caso l'integrale di contorno e il tasso di rilascio di energia unitario ottenuto mediante la subroutine esterna continuano ad avere andamenti pressoché identici anche per propagazioni molto maggiori. Si ritiene quindi valida la metodologia numerica sviluppata per il calcolo di $G$.

L'analisi viene svolta globalmente in quattro passi successivi:

i) nel primo passo non viene considerato alcun danneggiamento e si carica il giunto fino al carico massimo da utilizzare nella propagazione a fatica;

ii) nel secondo passo il giunto viene scaricato, assegnando contemporaneamente un valore $d=1$ al punto di integrazione più sollecitato nello step precedente, in modo da creare un avanzamento virtuale del difetto mediante il quale può essere calcolato il valore di $\Delta G$ iniziale per la simulazione dell'avanzamento a fatica del difetto.

iii) nuovo caricamento del giunto con calcolo dell'eventuale danno statico dovuto alla rampa di carico;

iv) simulazione dell'avanzamento a fatica del difetto in cui il carico viene mantenuto costante.

In quest'ultimo passo, ricavato il $\Delta G$ applicato si verifica che questo sia maggiore del limite di fatica $\Delta G_{t b}$. Se tale condizione è soddisfatta parte il loop per la previsione dell'avanzamento del difetto (Fig. 5). Per ogni punto di integrazione, conoscendo il danno $d$ accumulato fino a quel momento, viene calcolato il danno $\bar{d}$. Si impone quindi l'aumento di danno $\Delta \bar{d}$ e si calcola il nuovo valore $\overline{d^{\prime}}$ : questo sarà assunto pari a se stesso se risulta essere minore di 1 , 
oppure pari all'unità in caso contrario. Per ogni punto di integrazione si hanno quindi un valore iniziale e finale di $d$. Integrando l'Eq. 12 tra questi due valori si identifica un numero di cicli per ogni punto dei integrazione, e si assume come numero di cicli dell'incremento il minore tra tutti questi. Mediante questo numero di cicli si aggiorna il danno $d$ di ogni elemento integrando nuovamente l’Eq. 12, al fine però di trovare il nuovo valore di danno.

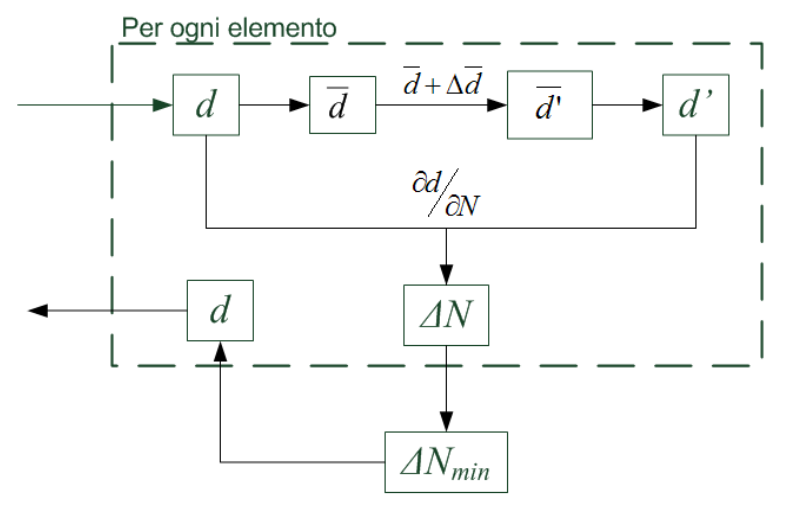

Figura 5: Schema di funzionamento della subroutine.

Questa operazione è quindi ripetuta fino all'incremento in cui il valore di $G$ raggiunge il valore critico $G_{C}$. Ad ogni incremento corrisponde quindi una sollecitazione $\Delta G$, un numero di cicli $\Delta N$, ed un avanzamento del difetto $\Delta A$ (calcolato come la somma delle aree danneggiate $A_{d}$ di ogni elemento).

I primi due step permettono anche la determinazione di $\delta_{t b}$. Questo viene assunto pari al valore massimo di apertura tra tutti i punti di integrazione dello strato di adesivo, quando il $\Delta G$ applicato eguaglia il valore di soglia $\Delta G_{t h}$ (vedi Fig. 6).

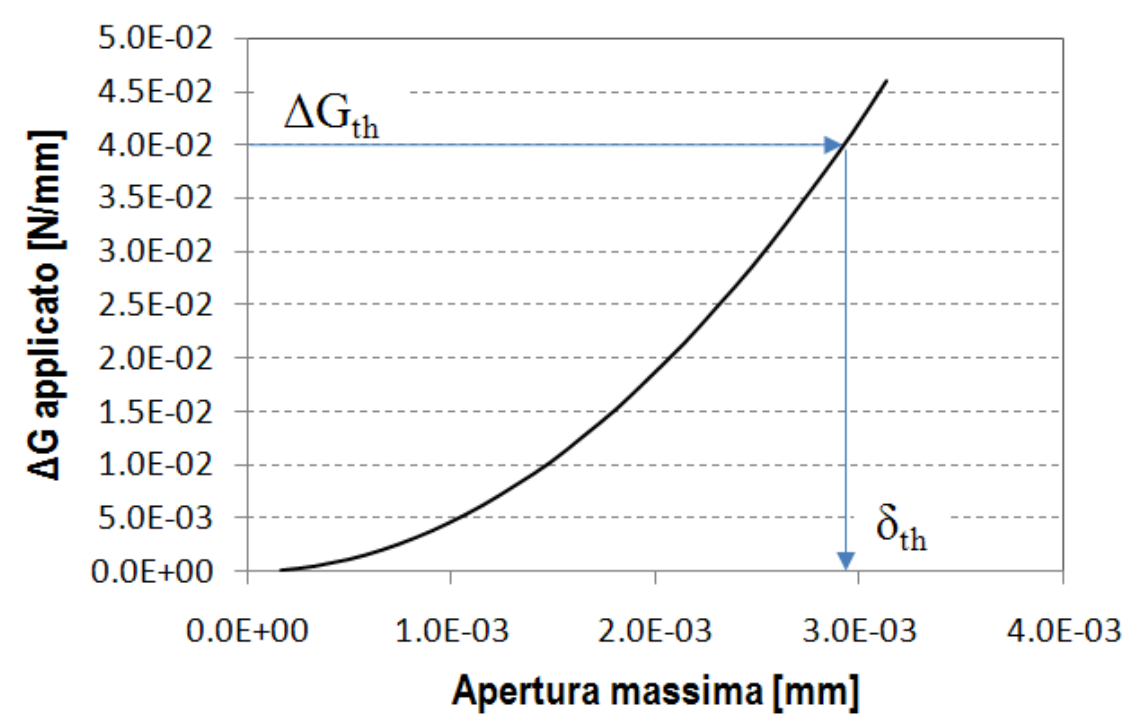

Figura 6: Determinazione del valore di $\delta_{\text {th. }}$.

\section{PARAMETRI DEL MODELLO}

$\mathrm{P}$ er lo svolgimento dell'analisi sono necessari i parametri della zona coesiva, cosi come i parametri della legge di Paris dell'adesivo. hanno portato alla definizione di una legge coesiva triangolare con le caratteristiche riportate in Tab. 1.

Per quel che riguarda invece i parametri della legge di Paris si sono ottenuti da prove di propagazione di difetti sempre su provini DCB [22]. In Tab. 2 sono riportati i coefficienti della regressione a legge di potenza (rapporto di carico $\mathrm{R}=0.1$ ). 


\begin{tabular}{cc}
\hline Parametro & Valore \\
$\Gamma[\mathrm{N} / \mathrm{mm}]$ & 0.6 \\
$\sigma_{\max }[\mathrm{MPa}]$ & 30 \\
$\delta_{0}[\mathrm{~mm}]$ & 0.004 \\
$\delta_{C}[\mathrm{~mm}]$ & 0.04 \\
\hline
\end{tabular}

Tabella 1: Parametri del modello di zona coesiva.

\begin{tabular}{cc}
\hline Parametro & Valore \\
C & 5.19 \\
$\mathrm{~m}$ & 3.64 \\
\hline
\end{tabular}

Tabella 2: Coefficienti della legge di Paris.

\section{RISULTATI E DISCUSSIONE}

A

1 fine di verificare la validità dell'implementazione i risultati della simulazione sono stati confrontati con i dati sperimentali [22]. I risultati mostrati in Fig. 7sono stato ottenuti con un incremento massimo del danno $\Delta \bar{d}$ pari a 0.05 per ogni incremento dell'analisi.

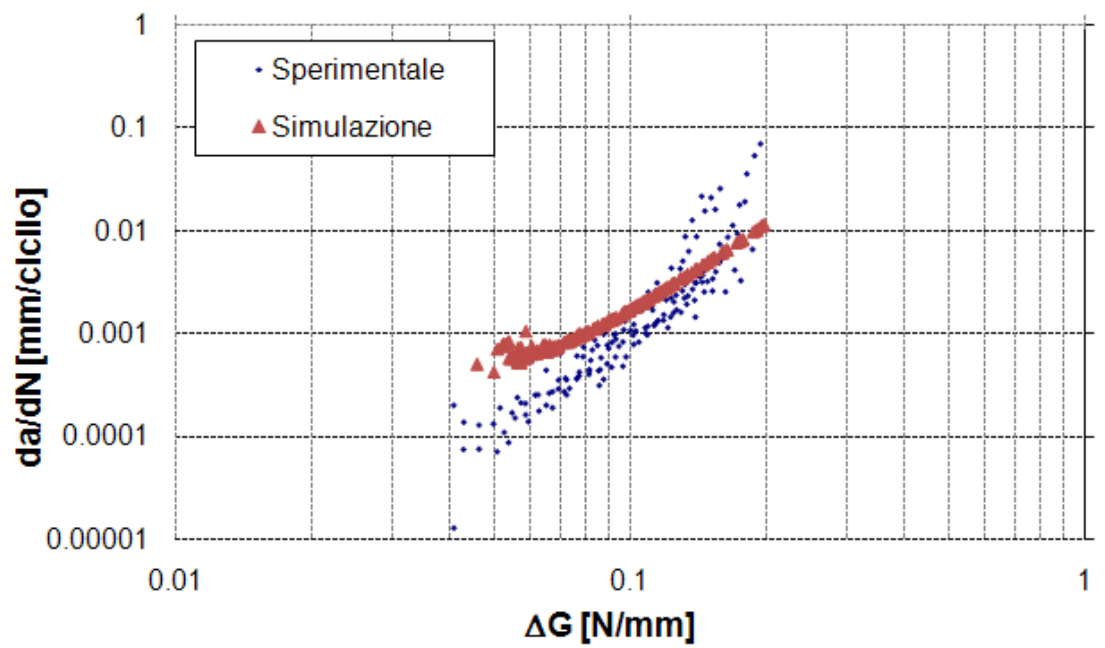

Figura 7: Confronto tra risultati sperimentali e previsione del modello ottenuto con Eq. 12.

Si nota come i risultati delle simulazioni rientrino nel campo di quelli ottenuti sperimentalmente anche se si può notare una pendenza inferiore. Tale differenza può viene evidenziata anche nei risultati delle analisi svolte in [12] (Fig. 8) dove la pendenza della curva ottenuta per via numerica è inferiore rispetto a quella ottenuta dalla regressione di dati sperimentali. Questo fenomeno si ritiene sia legato al criterio di omogeneizzazione del danno rappresentato dalle Eq. 4 e 10.

Esaminando l'Eq. (12) si può notare che, definito un rateo di avanzamento del difetto $d A / d N$, il rateo di aumento del danno per i punti di integrazione con danno pari 0 , sta in un rapporto circa pari a $\left(\delta_{f} / \delta_{0}\right)^{2}$ rispetto al rateo di aumento di danno dei punti di integrazione con danno prossimo a 1 . Nel caso in cui il valore di $\delta_{0}$ sia molto minore del valore di $\delta_{f}$ (e nel caso in esame c'è un rapporto di circa $1 / 10$ ) questo porta gli elementi lontani dall'apice del difetto a danneggiarsi molto più velocemente degli elementi prossimi all'apice stesso (nel caso in esame il rapporto è prossimo a 100). Legando invece il danno a livello microscopico ai suoi effetti sulla rigidezza [20], cioè

$$
d=\frac{A d}{A e}=1-\frac{K}{K_{0}}
$$


e svolgendo di nuovo tutti i passaggi mostrati nella sezione precedente si arriva ad un legame

$$
d d / d N=\frac{1}{A_{C Z}} d A / d N
$$

In questo legame non vi è alcuna dipendenza dal valore del danno, ovvero tutti gli elementi appartenenti alla zona di processo $A_{C Z}$ presentano il medesimo rateo di aumento del danno.

Svolgendo una analisi identica alla precedente in cui viene utilizzata l'Eq. (17) al posto della (12), si arriva al risultato mostrato in Fig. 9. Si nota come i punti della simulazione siano decisamente più allineati a quelli sperimentali rispetto a Fig. 7 , anche se la simulazione continua a sovrastimare la velocità di avanzamento nella fase iniziale dell'analisi.

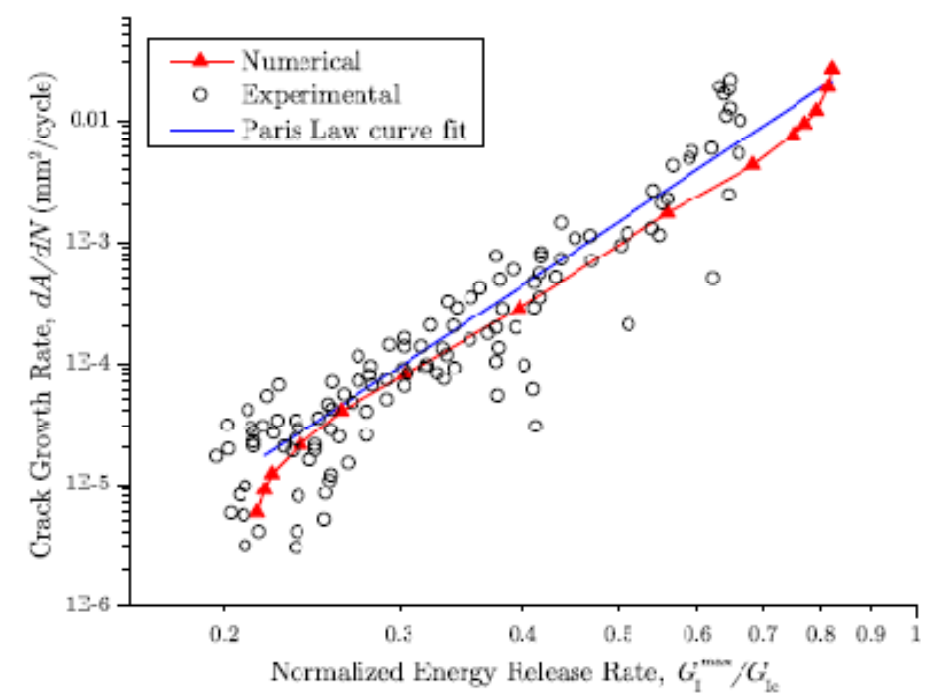

Figura 8: Confronto tra risultati sperimentali e previsione del modello da [12].

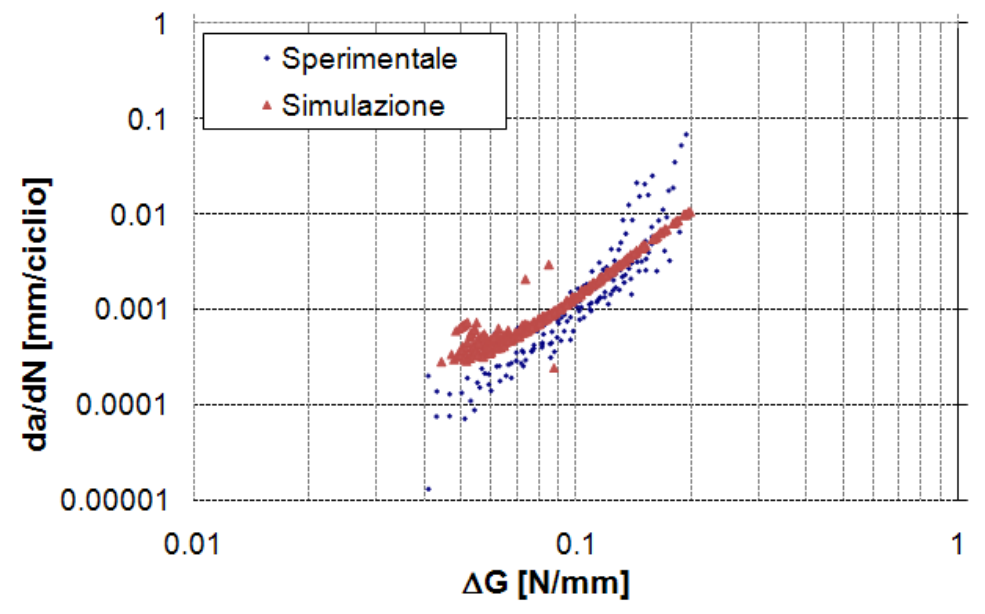

Figura 9: Confronto tra risultati sperimentali e previsione del modello ottenuto con Eq. (16).

Un ulteriore modifica, in fase di implementazione, è una legge di omogeneizzazione del danno diversa dalla semplice ripartizione in parti uguali dell'avanzamento del difetto sugli elementi compresi in $A_{C Z}$ rappresentata dall'Eq. (10).

$\mathrm{Si}$ è inoltre valutata l'influenza sul risultato della dimensione della mesh degli elementi coesivi. Fig. 10 mostra il valore del coefficiente $m$ della regressione ottenuta dai risultati delle analisi per varie dimensioni degli elementi coesivi. Per una maggiore chiarezza si sono normalizzati i dati rispetto ad una dimensione di $0.4 \mathrm{~mm}$ che è quella utilizzata per i precedenti risultati. 


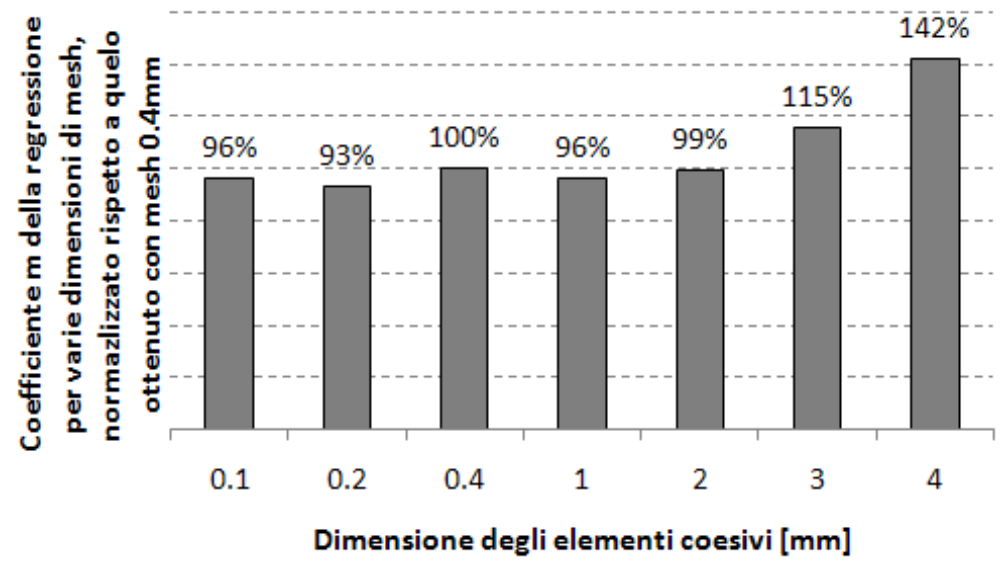

Figura 10: Influenza della dimensione della mesh sulla pendenza della regressione dei risultati delle simulazioni.

Si può notare come da mesh molto fitte $(0.01 \mathrm{~mm})$ fino a $2 \mathrm{~mm}$, non vi sia una grossa differenza dei risultati. Aumentando ancora la mesh si nota invece come i risultati si discostino sempre di più.

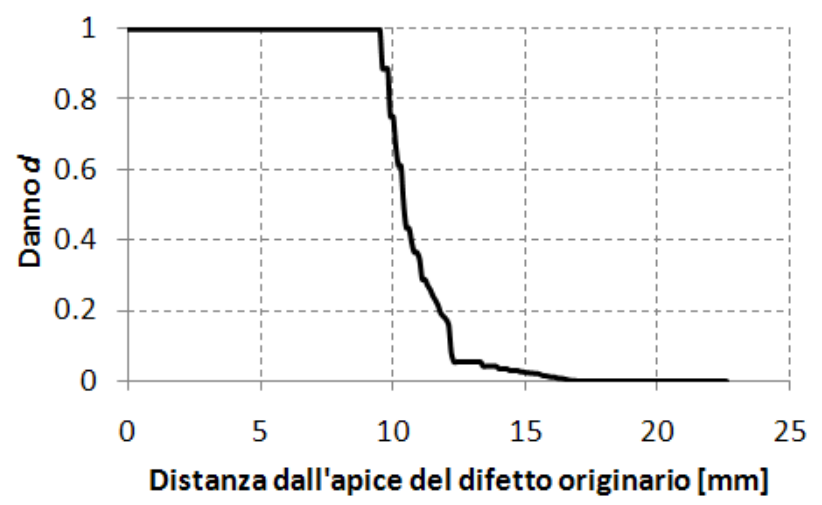

Figura 11: Distribuzione della variabile di danno d in funzione della distanza dall'apice del difetto.

Fig. 11 mostra l'andamento della variabile danno in funzione della distanza dell'apice del difetto originale, quando la zona coesiva è completamente sviluppata: questo mostra come la zona coesiva interessi una dimensione circa $8 \mathrm{~mm}$ Noto ciò e nota l'influenza della mesh sui risultati, si può quindi identificare un numero minimo di elementi che devono essere presenti all'interno della zona coesiva, quando questa è completamente sviluppata, pari a 4. Questo è necessario per permettere di descrivere in modo accurato le tensioni e i relativi spostamenti all'apice del difetto.

Si è infine valutata l'influenza di $\Delta \bar{d}$. Il grafico in Fig. 12 mostra come varia la vita a fatica di un giunto (la geometria è la medesima cui si riferisce Fig. 4) in funzione della variazione massima del danno.

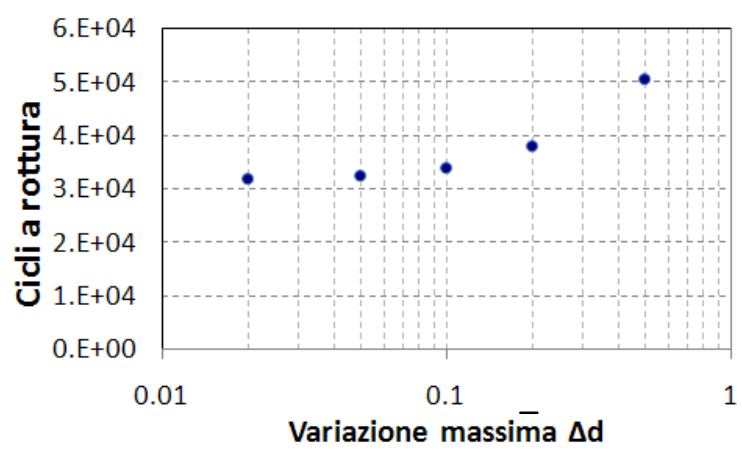

Figura 12: Influenza del valore di $\Delta \bar{d}$ sulla previsione di vita a fatica. 
Diminuendo tale limite da 0.5 fino ad un valore di 0.01 si nota come le durate vadano diminuendo asintoticamente. Questa differenza può essere spiegata analizzando l'Eq. (12), in cui la derivata del danno $d$ rispetto al numero di cicli è funzione del danno stesso. Di conseguenza tanto più sono piccoli gli intervalli su cui tale equazione viene integrata, tanto più accurati saranno i risultati. La diminuzione di $\Delta \bar{d}$ porta però a tempi di calcolo via via crescenti, ed al fine di ottimizzare la procedura è conveniente fare una sorta di analisi di convergenza cosi come viene di norma svolto per quanto riguarda la dimensione della mesh.

\section{CONCLUSIONI}

$\mathrm{S}$ i è eseguita l'implementazione di una procedura per la previsione della propagazione di difetti a fatica attraverso una subroutine esterna interagente con il software ABAQUS. In questo modo si è anche reso possibile il calcolo automatico dei valori della variazione del tasso di rilascio di energia applicato e della dimensione della zona di processo. La simulazione di propagazione di difetti in Modo I in giunti DCB, ha mostrato risultati caratterizzati da una pendenza dell'andamento della velocità di propagazione in funzione di $\Delta G$ inferiore a quello sperimentale, come riscontrato anche in [12]. Al fine di individuare il motivo di tale scostamento e cercare di limitarlo, viene proposta una nuova legge di omogeneizzazione del danno, che permette di ridurre le differenze, senza però eliminarle. Sviluppi futuri interesseranno l'identificazione di differenti leggi di omogeneizzazione del danno in modo da ripercorrere in modo più fedele i dati sperimentali. Inoltre il modello sarà esteso anche per sollecitazioni agenti in modo II in modo da poter ampliare la varietà di giunti analizzabili.

\section{BIBLIOGRAFIA}

[1] G. Barenblatt, Adv. Appl. Mech., 7 (1962) 55.

[2] D. Dugdale, J. Mech. Phys. Solids., 8 (1960) 100.

[3] I. Mohammed, K.M. Liechti, J. Mech. Phys. Solids, 48 (2000) 735.

[4] Q.D. Yang, M.D. Thouless, S. M. Ward, J. Mech. Phys. Solids, 47 (1999) 1337.

[5] W. G. Knauss, G. U. Losi, J. Appl. Mech., 60 (1993) 793.

[6] H. Hadavinia, A. J. Kinloch, J. G. Williams, In Adv. in Fract. and Damage Mech. II, (M. Guagliano, M.H. Aliabadi eds.), Hoggar, Geneva, (2001) 445.

[7] B. F. Sorensen, , Acta Mater., 50 (2002) 1053.

[8] B. F. Sorensen, , Jacobsen, T.K., Eng. Fract. Mech., 70 (2003) 1841.

[9] I. Georgiou, H. Hadavinia, A. Ivankovic, A.J. Kinloch, V. Tropsa, J.G. Williams, J. Adhesion, 79 (2003) 239.

[10] B.R.K. Blackman, H. Hadavinia, A.J. Kinloch, J.G. Williams, Int. J. Fract., 119 (2003) 25.

[11] T. Pardoen, T. Ferracin, C.M. Landis, F. Delannay, J. Mech. Phys. Solids, 53 (2005) 1951.

[12] Turon, Costa, Camanho, Dàvila, Composites, 38 (2007) 2270.

[13] K.L. Roe, T. Siegmund, Eng. Fract. Mech., 70 (2003) 209.

[14] S. Maiti, P.H. Geubelle, Eng. Fract. Mech., 72 (5) (2005) 691.

[15] A. Abdul-Baqi, P.J.G. Schreurs, M.G.D. Geers, Int. J. of Solids and Structures, 42 (2005) 927.

[16] J.J. Munoz, U. Galvanetto, P. Robinson, Int. J. Fatigue, 28 (2006) 1136.

[17] M. Erinc, P.J.G. Schreurs, M.G.D. Geers, International Journal of Solids and Structures, 44 (2007) 5680.

[18] P. Paris, F. Erdogan, J. Basic Eng 85 (1961) 528.

[19] S. Krenk, Eng. Fracture Mech. 43 (1992) 549.

[20] J. Lemaitre, J. of Engn. Mat. and Tech., 107 (1985) 83.

[21] A. Pirondi, D. Fersini, E. Perotti, F. Moroni, Atti del XIX Convegno Nazionale IGF, Milano (2007).

[22] A. Pirondi, F. Moroni, M. Vettori, Atti del XXXVI Convegno Nazionale AIAS, Ischia (2007). 